\title{
KAL-HAITI: A RESEARCH DATABASE FOR RISKS MANAGEMENT AND SUSTAINABLE RECONSTRUCTION IN HAITI
}

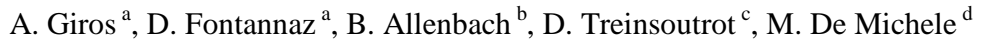 \\ ${ }^{a}$ CNES, 18 av. Edouard Belin, 31400 Toulouse, France - (alain.giros, delphine.fontannaz)@cnes.fr \\ ${ }^{\mathrm{b}}$ SERTIT, bd Sébastien Brant, 67412 Illkirch, France - bernard.allenbach@ sertit1.u-strasbg.fr \\ ${ }^{c}$ CETE-SO, av Colonel Roche, 31400 Toulouse, France - Didier.Treinsoutrot@ developpement-durable.gouv.fr \\ ${ }^{\mathrm{d}}$ BRGM, Av C. Guillemin, 45060 Orléans, France - m.demichele@ brgm.fr
}

KEY WORDS: Risks Management, Database, Data quality, High resolution images, Research support

\begin{abstract}
:
Following the 12th January 2010 earthquake in Haiti, the French Agence Nationale de la Recherche has funded a project named KAL-Haiti which aims at gathering remote sensing imagery as well as in-situ and exogenous data into a knowledge base. This database, seen as a shareable resource, can serve as a basis for helping the reconstruction of the country, but also as a reference for scientific studies devoted to all phases of risk management. The project main outcome will be a geo-referenced database containing a selection of remotely sensed imagery acquired before and after the disastrous event supplemented with all relevant ancillary data, and enriched with in-situ measurements and exogenous data. The resulting reference database is freely available for research and for reconstruction tasks. It is strongly expected that users will also become contributors by sharing their own data production, thus participating to the growth of the initial kernel. The database will also be enriched with new satellite images, monitoring the evolution of the Haitian situation over the next 10 years.
\end{abstract}

\section{INTRODUCTION}

The earthquake that struck Haiti in January 2010 is amongst the most destructive disasters of recent years: more than 200000 lives lost, tens of thousands injured, close to 2 million people made homeless, the entire country must be rebuilt. The response of the international community has been unprecedented. Over and above the emotion brought on by the terrible scenes in the aftermath of the earthquake, our focus is now turned to rebuilding the country. In addition, using the abundance of data available, this case must be studied with the aim of better understanding seismic hazard and vulnerability in this zone and elsewhere.

During the immediate response to the disaster, tens of satellite images, both optical and radar, covering various spatial resolutions were acquired by satellite operators whether they be national space agencies or private companies. To these were added aerial photography and in situ measurements. After photo-interpretation, these images were used by the civil protection agencies that sent teams to the scene.

Too often limited to this « response » phase, this earth observation and in situ data should be available for use by relevant agencies involved throughout the disaster management cycle : from recovery, to mitigation, to preparedness. A prerequisite for making all this data available (in situ measurements, images, maps, models, reports, statistical data) to users (decision makers for the reconstruction effort, scientists) must be that it is accessible in an easily exploitable form (geo-referenced, corrected, validated and dated).

This paper presents the KAL-Haiti project which has been setup in order to propose a new resource freely accessible by researchers, developers and final users, for developing and evaluating new solutions in the disaster management cycle.

\section{MOTIVATIONS}

Researchers and solution developers must exhibit extensive know-how and a good dose of creativity in order to devise innovative solutions to the complex problems they try to solve. However, this is not sufficient. Firstly it is better when the problems correspond to real issues and are defined by the potential users, either directly or indirectly, and secondly well documented and qualified datasets must be available and accessible in order to design and validate the proposed solutions.

Taking into account the humanitarian importance of the Haiti earthquake catastrophe and the strong demand for helping reconstruction, as well as the huge amount of available data related to this situation and the complexity of this crisis management, the KAL-Haiti project's principal aim is thus to produce and promote the use of a database of earth observation (remote sensing and in situ) and associated data concerning the Haiti earthquake, defined and used jointly by end users and scientists.

The reference infrastructure that is setup by the project, containing freely exploitable data, provides an invaluable resource for research into developing methods of analysis for users involved in disaster management from pre-event preparedness to the return to normal after the event, but also during the reconstruction phase currently underway in Haiti.

This project is aligned with current activities in the partner organisations. These include the CNES ISIS and KALIDEOS programmes which provides improved access for the science and applications community to SPOT imagery, and CNES' participation and role as project manager for the Haiti disaster for the International Charter on Space and Major Disasters. 


\section{PROJECT OVERVIEW}

The KAL-Haiti project can be described as four different components which interact with each other. These four components correspond to the main concerns which are considered for a successful use of this resource:

- A community of contributors for a joint involvement of users and scientists,

- A data procurement and processing entity, in charge of the quality and availability of the data,

- The database itself which exposes the data to public and registered users,

- The promotion and follow-up of applications of the database..

These elements are described in the following subsections and their interactions are shown in figure 1.

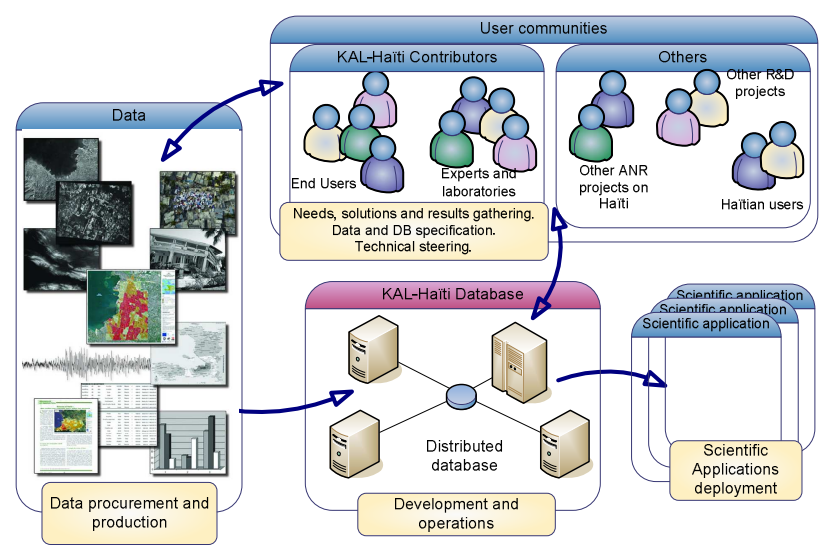

Figure 1 - KAL-Haiti components and interactions

\subsection{Community of contributors}

One of the characteristics of this project is the strong user involvement, whether they be, for instance, involved in humanitarian aid (Red Cross), UN organisation ( UNITAR/UNOSAT) or specialists in urban planning.

Starting from January 2011, the project team has recruited several of these organisations acting in Haiti and also research institutes specialized in data analysis, geophysics or risks management. A call for contribution has been issued based on the win/win principle: the contributors give their expertise and express their needs which are in turn taken into account in order to optimize the definition, the content and the validation process of the KAL-Haiti database. This community will be active at least during the development of the project (2010-2014), and has been structured around several thematic groups: risks management, urban planning, geology, seismology, data processing.

Communication within the community of contributors is facilitated by a newsletter, a website and workshops. These workshops have been organized since 2011 and are the main place for contributors to exchange information on available data and existing or new coming applications, express needs in satellite and exogenous data related to the Haiti catastrophe, and identify new contributors. A major outcome of these workshops is the identification of new applications which can be carried out within the project framework. A short list of such applications identified in the 2011 workshop is given in table 1 . Next workshops will be focused on the assessment of the results of selected applications and the definition of future activities.

\begin{tabular}{|l|l|}
\hline \multicolumn{1}{|c|}{ Application } & \multicolumn{1}{|c|}{ Study area } \\
\hline Follow-up of refugee camps & Port - au - Prince \\
\hline Planning of urban districts & $\begin{array}{l}\text { Martissant (district in } \\
\text { Port - au - Prince) }\end{array}$ \\
\hline $\begin{array}{l}\text { Development of a database to } \\
\text { improve territorial management }\end{array}$ & $\begin{array}{l}\text { Jacmel (town south of } \\
\text { Port - au - Prince) }\end{array}$ \\
\hline Management of the risk of flood & Port - au - Prince \\
\hline Modeling of the risk of runoff & Port - au - Prince \\
\hline Seismic micro-zoning & Port - au - Prince \\
\hline \begin{tabular}{l} 
Atlas of natural risks \\
\hline $\begin{array}{l}\text { Cartography and prioritization } \\
\text { of the active faults: application } \\
\text { to risks assessment }\end{array}$
\end{tabular} & $\begin{array}{l}\text { South-East of Haiti } \\
\text { (Enriquillo peninsula) }\end{array}$ \\
\hline
\end{tabular}

Table 1. Some of the applications of the KAL-Haiti database considered so far.

\subsection{Data procurement and processing}

Due to the wide spread of information technologies, information has become more and more available. But in crisis situations like the one which followed the Haiti earthquake, information flows may present a chaotic behaviour: they are produced through different initiatives, acquired by different sensors and means, broadcasted through uncorrelated channels, stored in separate systems and used in independent contexts. One may argue that this behaviour is a normal consequence of a lack of preparation in crisis management, which is very difficult for events of such extents. As a result this huge amount of spread information can be a problem for the researchers and for those trying to help for the recovery. In order to access it in a sensible way, one must know which information is available, at what time, with what level of quality and be sure that it is interoperable with other information flows.

\subsubsection{Data inventory, gathering and procurement}

Basing scientific and technical activities upon a set of well identified information sources is very important. This is a prerequisite for good science where reproducibility is always a concern. This is also important for decision makers who need reliable information. Therefore the KAL-Haiti project has setup an inventory of the information sources which can be considered for its activities. This inventory includes satellite and aerial imagery but not only. It also contains the cartographic material produced by a number of agencies or communities, the damage assessments made by different authorities (e.g. PDNA), models designed by scientists from different disciplines, in-situ measurements, and so on. The main criteria used for selecting a source to be included in the inventory is the plausibility that it may be considered as a reliable source of information by some of the project contributors. Depending on the case the data is then collected from external sources or purchased through the best suited commercial channel, or even created if not available somewhere. 


\subsubsection{Data quality assessment and improvement}

Even if some data has been selected for inclusion in the database, it is not always of the best possible quality. Within the resources allocated to the KAL-Haiti project, we thus strive to apply the state-of-the-art data processing methods in order to 1) assess the data quality level 2) raise this quality level using the best of our know-how, if necessary. This is required by the researchers who, most often, are unwilling to bear the burden of correcting data of poor quality. Some examples of this processing are: denoising, radiometric calibration, reflectance computation, geometric accuracy.

\subsubsection{Data interoperability}

In crisis management situations, information is produced with different modalities (e.g. optical and SAR imagery) and in different formats or reference systems. However none of these information sources alone is sufficient to face the complexity of the problems posed by the situation. As a consequence, fusion of information coming from different sources is necessary, provided that these data are interoperable. The KAL-Haiti project takes this requirement into account by building data whose properties are consistent throughout the whole dataset: dating, geo-referencing, ortho-rectification, physical meaning, consistent metadata, standard nomenclatures and formats, etc.

\subsubsection{Data accessibility}

One important point for efficient research as well as effective support to humanitarian initiatives is the free accessibility to the data. This is natural for data whose license grants to everybody a free right to use them. But this is a concern for data which are normally distributed within a commercial framework because there exist no suitable generic license allowing non-commercial uses in specific domains and for specific activities. Thus the access to these commercial data in the KAL-Haiti database is granted only to registered users on a per data basis, allowing the traceability of their non-commercial uses.

\subsection{The database}

The datasets produced within the KAL-Haiti project are stored into a database which is accessible through Internet (KAL-Haiti website). The solution used so far for this implementation is based on the KALIDEOS solution, and allows to query the database using a small number of criteria (area of interest, data type, sensor, etc.) and to retrieve the files containing the data.

In the second part of the project a solution built on more cutting-edge technologies will be designed, allowing the users to browse, combine, amend and enrich the datasets online.

\subsection{Applications promotion and follow-up}

Once they have been selected together with the community of contributors, new applications are launched by mobilizing either project or external resources. The goal is to propose, for each problem which has been identified, the best possible solution involving end-users with real needs in Haiti and the highest-level scientific laboratories. Involvement of Haitian teams or institutes is also considered in order to favour spin-off on these topics.

The selected applications are then followed up as usual scientific and technical developments, except that their outcome (progress, produced data, publications, models, etc) are systematically provided to the database.

\section{EXAMPLES OF APPLICATIONS}

In this section three applications which have been conducted (first and second ones) or which could be realized (third one) are briefly presented. They illustrate the diversity of the needs and problems and also the richness of the database results. More applications results will be available on the KAL-Haiti website as soon as they become available and will be shown during the oral presentation.

\subsection{Reconstruction monitoring in urban areas}

Construction and reconstruction of safe houses and buildings after such a major disaster as the January,12 2010 earthquake is one of the major challenges facing the Haitian nation and the international community. Indeed a lot of initiatives have been carried out: hard shelters have been installed in many locations, destroyed buildings have been removed in most cities, houses repairs have been made, and new buildings have been erected.

Monitoring this rapidly evolving situation is thus very important from different perspectives: social services, economy planning, health care, to name a few. In order to be effective, such a monitoring should cover wide areas in order to account for the whole population of the cities and should be updated at short intervals for near real-time awareness. Remote sensing imagery can thus be very useful for monitoring such evolutions in this kind of situation.

In order to assess the added value of high resolution remote sensing imageries for such situation monitoring, the KAL-Haiti project has produced a set of vector layers built from satellite and aerial imagery, and showing the buildings footprints at different periods. Three layers showing the location and status of 22257 buildings over the Jacmel area $\left(50 \mathrm{~km}^{2}\right)$ have been produced by photo-interpretation: one from a Worlview-1 image acquired on Dec. 9, 2009, before the earthquake, a second one just after the earthquake from the NOAA aerial campaign made on Jan. 24, 2010 and a third one 18 months later using a Worldview-2 image acquired on July 17, 2011.

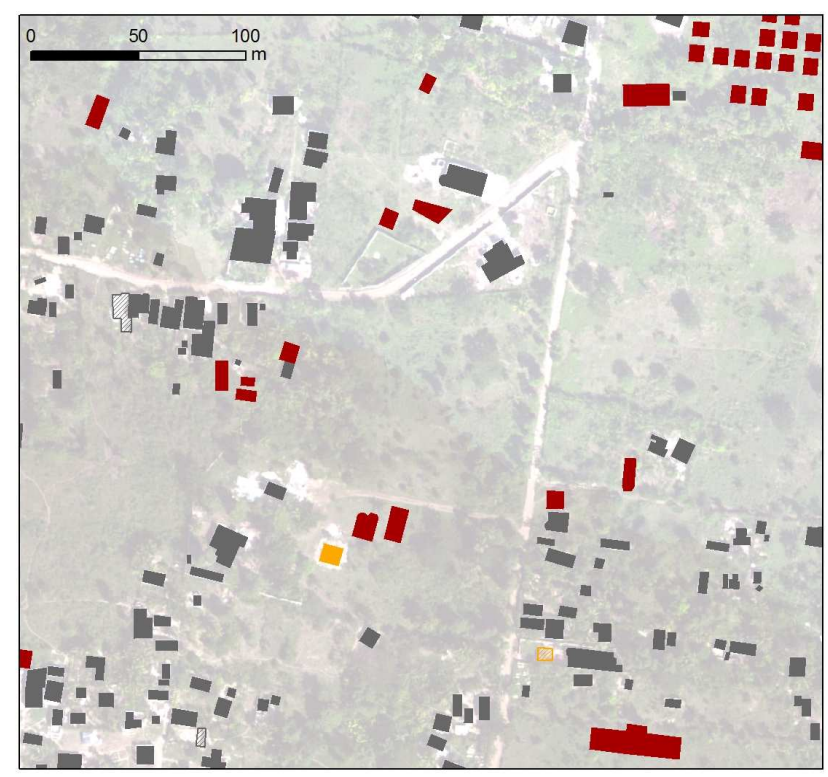

Figure 2 - Evolution of buildings and shelters in Jacmel area, at 3 dates: before, just after and 18 months after the earthquake. 
By comparison of these different layers, an enriched layer allowing identification of the evolutions of buildings, houses and settlements can be created. An excerpt of this vector layer showing the status of buildings and houses in a small area of Jacmel is shown in figure 2. Buildings existing before the earthquake are shown as grey boxes, either opaque if they still exist or empty if they have been removed. Buildings built in the emergency period are displayed as orange boxes, either opaque if they still exist or empty if they have been removed since then. Finally red boxes correspond to new buildings or shelters built since the emergency period. One must be aware that a dark grey box does not always correspond to a safe building, because this layer does not take into account the safety status of the building (available in another layer). However this information can be very useful for the Jacmel authorities in their effort to plan the city reconstruction.

\subsection{Analysis of water runoff in urban area}

Water runoff is a major risk in several districts of Port-auPrince and its impact may be higher than usual, given the habitat conditions in this area after the disaster. Assessing this risk and associated vulnerability is thus a major challenge for Haiti.

Information extracted from satellite imagery like buildings, roads and paths, together with a DEM (possibly derived from the same imagery) can be used to compute the water paths. Each building vulnerability can also be derived by merging these water paths and the building locations, geometry and status. This is shown in fig 3 for Martissant district. Furthermore, the same spatial description of the scene can be fed into hydrological models to estimate the water runoff at each location; improving the fitting of such models could become the subject of subsequent research activities.

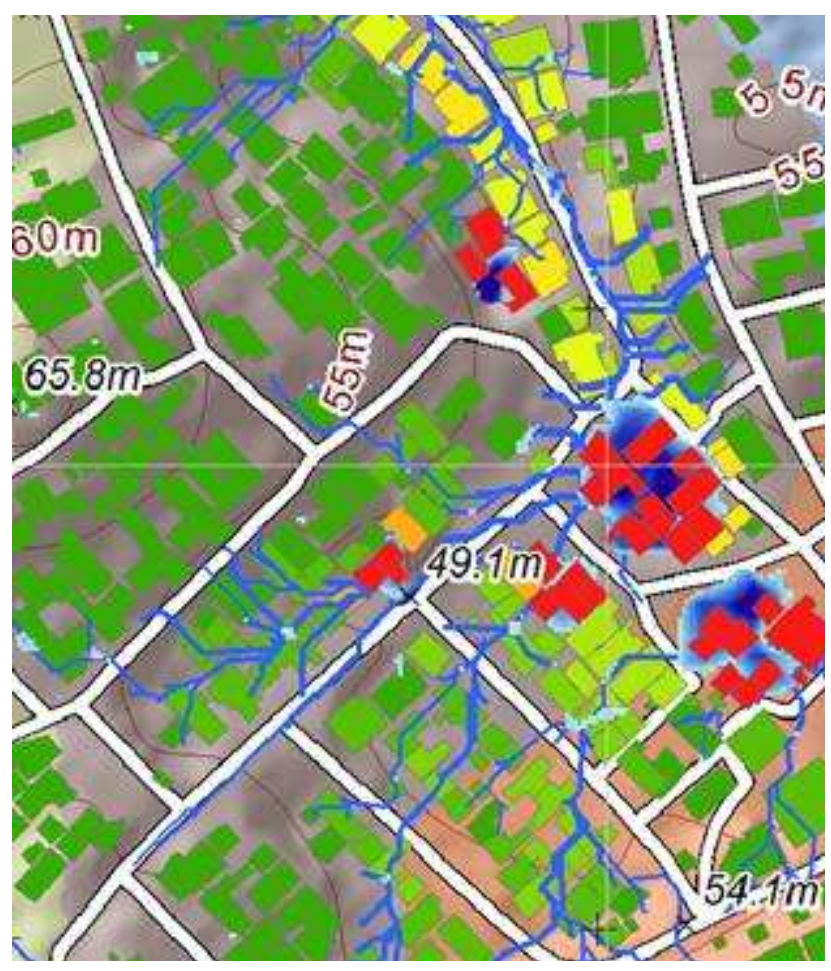

Figure 3 - Water runoff risk assessment showing the water paths and the building vulnerability.

\subsection{Automated reconstruction monitoring}

Vector layers described in section 4.1 can also be used, together with the high resolution images they come from, as a test case for developing new (semi-)automated methods aiming at identifying buildings evolutions in high resolution images, taking into account the buildings layers when they already exist. In situations where housings are rapidly evolving, such methods could be very effective because they could help reducing the delay for such information production.

\section{PERSPECTIVES}

Besides the applications given as examples in the previous section, KAL-Haiti steadily promotes the proposal of new applications and research activities in the field of global risk management and sustainable reconstruction, from geophysical and societal modelling to image analysis, data processing and information management.

From another perspective, the CNES KALIDEOS programme, in which KAL-Haiti is inscribed, is committed to support such databases and surrounding activities on the long term, thus providing a stable environment for contributors willing to be involved.

The KAL-Haiti database will also be proposed as a contribution to the Global Earth Observation System of Systems (GEOSS) « Supersites » activity which has begun to bring together relevant data for scientific study of this event

Considered together, these three arguments shall strengthen the potentialities of the database on the long term..

Last but not least, Haiti will of course benefit directly from the KAL-Haiti project. A mirror database will be transferred to Haiti during the course of the project and assistance in exploiting this resource will be proposed with the aim of developing a regularly updated GIS, operated by academic or institutional Haitian bodies.

\section{CONCLUSION}

The availability of comprehensive and consistent datasets corresponding to real use cases is a key issue for the development of new methods and algorithms able to solve increasingly complex problems. In line with this assumption, the KAL-Haiti database is a promising initiative which strives to strengthen the global risk management domain which has emerged as an important one, given its societal importance.

\section{REFERENCES}

ANR CFP: www.agence-nationale-recherche.fr/AAP-310Flash-Haiti.html (16/04/2012)

KAL-Haiti website: http://kal-haiti.kalimsat.fr, (16/04/2012)

\section{ACKNOWLEDGEMENTS}

The KAL-Haiti project is funded by the French Agence Nationale de la Recherche (ANR) under grant number 2010 HAIT 008 01. Contributions of data providers to the database, whose list is available on the Kal-Haiti website, are acknowledged by the KAL-Haiti project team. 